\title{
ON THE LINEAR SPAN OF LATTICE POINTS IN A PARALLELEPIPED
}

\author{
MARCEL CELAYA
}

\begin{abstract}
Let $\Lambda \subset \mathbf{R}^{n}$ be a lattice which contains the integer lattice $\mathbf{Z}^{n}$. We characterize the space of linear functions $\mathbf{R}^{n} \rightarrow \mathbf{R}$ which vanish on the lattice points of $\Lambda$ lying in the half-open unit cube $[0,1)^{n}$. We also find an explicit formula for the dimension of the linear span of $\Lambda \cap[0,1)^{n}$. The results in this paper generalize and are based on the Terminal Lemma of Reid, which is in turn based upon earlier work of Morrison and Stevens on the classification of four dimensional isolated Gorenstein terminal cyclic quotient singularities.
\end{abstract}

\section{INTRODUCTION}

Let $n$ be a positive integer and let $\Lambda$ denote a lattice in $\mathbf{R}^{n}$ that contains the integer lattice $\mathbf{Z}^{n}$. We are interested in understanding the combinatorics of the lattice points of $\Lambda$ inside the half-open cube

$$
[0,1)^{n}:=\left\{\left(x_{1}, \ldots, x_{n}\right) \in \mathbf{R}^{n}: 0 \leq x_{i}<1 \text { for all } i=1,2, \ldots, n\right\} .
$$

In general, questions about these points are difficult. For instance, if $\Lambda=\frac{1}{2} \mathbf{Z}^{n}$ and $u=\left(u_{1}, \ldots, u_{n}\right) \in \mathbf{R}^{n}$ has integral coordinates, then the problem of deciding if there exists a nonzero point in $\Lambda \cap[0,1)^{n}$ on the hyperplane $\left\{x \in \mathbf{R}^{n}:\langle u, x\rangle=0\right\}$ is NP-complete. Indeed, it is straightforward to reduce SUBSET-SUM to this problem; such a point exists if and only if some integers in the multiset $\left\{u_{1}, \ldots, u_{n}\right\}$ sum to zero. As pointed out by Sebö in [17, p. 401], the well-known Lonely Runner Conjecture [5] can be stated as a problem about the existence of a lattice point in $\Lambda \cap[0,1)^{n}$ satisying certain linear inequalities where the lattice $\Lambda$ is generated by $\mathbf{Z}^{n}$ plus a rational vector $v \in \mathbf{R}^{n}$ encoding the speeds of the runners.

Our approach to understanding the lattice points in $\Lambda \cap[0,1)^{n}$ begins with a result that is commonly attributed to G. K. White [19] but was discovered independently by several others [14, 16]. It says that a

2000 Mathematics Subject Classification. 52B20, 52B05, 11M20.

Research supported in part by the Natural Sciences and Engineering Research Council of Canada. 
tetrahedron $T$ in $\mathbf{R}^{3}$ which has integral vertices but no other integral points must be "sandwiched" between two parallel lattice hyperplanes. More precisely, there exists an integral normal vector $u=\left(u_{1}, u_{2}, u_{3}\right)$ and an integer $\delta$ such that two of the vertices of $T$ lie on the plane $\langle u, x\rangle=\delta$ and the other two lie on the plane $\langle u, x\rangle=\delta+1$. We may assume that one of the vertices of the tetrahedron lies at the origin, so that there are three integral vectors $v, v^{\prime}, v^{\prime \prime}$ corresponding to the three edges of the tetrahedron incident to the origin. The tricky part of White's theorem is to show that, after applying a unimodular transformation (i.e. a linear transformation of $\mathbf{R}^{n}$ which fixes $\mathbf{Z}^{n}$ ), we may further assume that $v=(1,0,0), v^{\prime}=(0,1,0)$, and $v^{\prime \prime}=(1, a, r)$ where $a$ and $r$ are positive integers such that $a<r$ and $a$ is coprime to $r$. From there, the normal vector $u=(1,0,0)$ establishes the conclusion of the theorem.

The triples $\lambda=\left(\lambda_{1}, \lambda_{2}, \lambda_{3}\right) \in \mathbf{R}^{3}$ such that $\lambda_{1} v+\lambda_{2} v^{\prime}+\lambda_{3} v^{\prime \prime} \in \mathbf{Z}^{3}$ form a lattice $\Lambda \subset \mathbf{R}^{3}$ which contains the integer lattice $\mathbf{Z}^{3}$. Moreover, $T$ contains a non-vertex integral point if and only if there exists some nonzero $\lambda \in \Lambda \cap[0,1)^{3}$ such that $\lambda_{1}+\lambda_{2}+\lambda_{3} \leq 1$. Indeed, such a $\lambda$ corresponds to a proper convex combination of at least two vertices of $T$. A short exercise shows that there are exactly $r$ lattice points in $\Lambda \cap[0,1)^{3}$ and they are of the form $(\{k a / r\},\{-k a / r\},\{k / r\})$ for $k=0,1,2, \ldots, d-1$. Here $\{x\}$ denotes the fractional part of the real number $x$, the unique real number in $[0,1)$ congruent to $x \bmod 1$. In particular, we can view the emptiness of $T$ as a consequence of the fact that the first two components of every nonzero $\lambda \in \Lambda \cap[0,1)^{3}$ sum to 1 and therefore the sum $\lambda_{1}+\lambda_{2}+\lambda_{3}$ exceeds 1 .

More generally, if $\Lambda \subset \mathbf{R}^{n}$ is a lattice that contains $\mathbf{Z}^{n}$, then we can think of the presence of such complementary pairs of coordinates as a restriction on the extent to which the nonzero points in $\Lambda \cap[0,1)^{n}$ can deviate from the hyperplane $x_{1}+\cdots+x_{n}=n / 2$. Sebö asks in [17] about the most restrictive case, where all the nonzero lattice points in $\Lambda \cap[0,1)^{n}$ lie on this hyperplane. He conjectures that this can only happen if the coordinates can be grouped into $n / 2$ pairs of complementary coordinates as above. More precisely, suppose $\Lambda$ is a lattice in $\mathbf{R}^{n}$ generated by $\mathbf{Z}^{n}$ and the point $\frac{1}{r}\left(a_{1}, \ldots, a_{n}\right)$, where the $a_{i}$ 's are positive integers coprime to a positive integer $r$. Note that for every $\left(\lambda_{1}, \ldots, \lambda_{n}\right) \in \Lambda \cap[0,1)^{n}$, there exists an integer $0 \leq k<r$ such that $\lambda_{i}=\left\{k a_{i} / r\right\}$ for each $i=1,2, \ldots, n$. Sebő asks if the following statement is true:

Conjecture 1.1. The equality

$$
\lambda_{1}+\lambda_{2}+\cdots+\lambda_{n}=n / 2
$$


holds for all nonzero $\lambda \in \Lambda \cap[0,1)^{n}$ if and only if $n$ is even and (after possibly reordering) $a_{i}+a_{i+1}=r$ for $i=1,3,5, \ldots, n-1$.

In [17], Sebö proves the case $n=4$ of his conjecture and uses it to deduce White's theorem.

It turns out, however, that Sebő's conjecture had already been established some years earlier by Morrison and Stevens in their paper [13] (see also [2]). Although they were also primarily interested in the case $n=4$, their proof stands out as it easily extends to all positive even integers $n$. In [13], Morrison and Stevens use this result to derive a complete classification of three dimensional isolated terminal cyclic quotient singularities and four dimensional isolated Gorenstein terminal cyclic quotient singularities. The survey paper of Borisov [6] provides a nice description and some interesting number-theoretic applications of this problem.

In [15, Theorem 5.4], Reid proves a stronger version of Conjecture 1.1 that does not require the $a_{i}$ 's to be coprime to $r$. Given a lattice $\Lambda \subset \mathbf{R}^{n}$ generated by $\mathbf{Z}^{n}$ and a point $\frac{1}{r}\left(a_{1}, \ldots, a_{n}\right)$ where the $a_{i}$ 's are positive integers less then $r$, he finds a characterization for when all lattice points in $\Lambda \cap[0,1)^{n}$ lie in a given hyperplane through the origin. We show in Section 3 how to deduce Sebő's conjecture from Reid's result, known as the Terminal Lemma. In [15, Section 6], Reid shows how the Terminal Lemma can be systematically applied to obtain Mori's classification results on three dimensional terminal singularities found in [12].

Other variations of Conjecture 1.1 have found application in Ehrhart theory; in particular the problem of classifying lattice polytopes with a given $h^{*}$-polynomial. In [3], Batyrev and Hofscheier give a classification of all lattice polytopes whose $h^{*}$-polynomial is of the form $h^{*}(t)=1+c t^{k}$ for some positive integers $c, k$ in terms of particular linear codes. This work was further developed by Higashitani, Nill, and Tsuchiya in [9] in order to obtain a combinatorial description of Gorenstein polytopes with a trinomial $h^{*}$-polynomial. A key ingredient of these results is a version of Conjecture 1.1 applicable to lattices $\Lambda \subset \mathbf{R}^{n}$ containing $\mathbf{Z}^{n}$ with the property that the quotient group $\Lambda / \mathbf{Z}^{n}$ is isomorphic to the additive group of a finite field.

In this paper, we establish of a variation of Conjecture 1.1 which imposes no restrictions on the lattice $\Lambda \subset \mathbf{R}^{n}$ except that it must contain the integer lattice $\mathbf{Z}^{n}$. The paper is organized into six sections. Following the introduction, Section 2 outlines the basic notation and concepts we use. In Section 3, we state our main theorem, which directly generalizes Reid's Terminal Lemma by dropping the assumption 
$\Lambda / \mathbf{Z}^{n}$ must be cyclic. From our theorem we deduce a formula for the dimension of the linear span of the points in $\Lambda \cap[0,1)^{n}$. We also state a natural generalization of Conjecture 1.1 when there are no assumptions on the group structure of $\Lambda / \mathbf{Z}^{n}$. Finally, we state the two main technical tools needed to prove our main theorem. Section 4 outlines the proof of our main theorem using these two tools, both of which are statements about an arbitrary additive finite abelian group $G$. Section 5 contains the proof of the first technical tool, which asserts that a specific collection of indicator functions defined on $G$ is linearly independent. In Section [6 is the proof of the second technical tool, which gives a specific spanning set for the space of functions $f: G \rightarrow \mathbf{C}$ satisfying $f(-a)=-f(a)$ for all $a \in G$. At a high level, we mostly follow the path laid out by Reid in [15]. We differ somewhat in the details, however, by making liberal use of the results in [11, Section 9.2].

\section{BACKGROUND AND NOTATION}

2.1. Notation. For $u=\left(u_{1}, \ldots, u_{n}\right) \in \mathbf{R}^{n}$ and $v=\left(v_{1}, \ldots, v_{n}\right) \in \mathbf{R}^{n}$, we let $\langle u, v\rangle=u_{1} v_{1}+\cdots+u_{n} v_{n}$ denote the usual dot product. If $v$ is a vector in a vector space with specified coordinates, then $\operatorname{supp}(v)$ denotes the set of coordinates $i$ for which $v_{i} \neq 0$. For $x \in \mathbf{R}$, we define $\{x\}$ to be be the unique real number in the half-open interval $[0,1)$ in which $x-\{x\}$ is an integer. We frequently make use of the fact that for any $x \in \mathbf{R},\{x\}+\{1-x\}$ equals 1 if $x \notin \mathbf{Z}$ and 0 otherwise. We define the first periodic Bernoulli function $B_{1}: \mathbf{R} \rightarrow \mathbf{R}$ by

$$
B_{1}(x):= \begin{cases}\{x\}-1 / 2, & x \notin \mathbf{Z} \\ 0, & x \in \mathbf{Z} .\end{cases}
$$

For $x+\mathbf{Z} \in \mathbf{R} / \mathbf{Z}$, we also define $B_{1}(x+\mathbf{Z}):=B_{1}(x)$.

For a finite group $G$, we denote the space of complex functions $f$ : $G \rightarrow \mathbf{C}$ by $L^{2}(G)$ which forms a vector space under pointwise addition and comes with the inner product

$$
\langle f, h\rangle=\frac{1}{|G|} \sum_{g \in G} f(g) \overline{h(g)} .
$$

2.2. Character theory of finite abelian groups. We refer the reader to [7] and [1] for an introduction to the character theory of finite abelian groups, and record some key facts here. For a finite abelian group $G$, let $\widehat{G}$ denote the multiplicative group of homomorphisms $G \rightarrow \mathbf{C}^{\times}$from $G$ to the nonzero complex numbers. The group operation of $\widehat{G}$ is given by pointwise multiplication: $(\chi \psi)(g):=\chi(g) \psi(g)$ for each $g \in G$ and for each $\chi, \psi \in \widehat{G}$. The inverse of $\chi \in \widehat{G}$ satisfies $\chi^{-1}(g)=\overline{\chi(g)}$ for 
all $g \in G$; we therefore denote $\chi^{-1}$ by $\bar{\chi}$. Elements in $\widehat{G}$ are called characters of $G$. There is an isomorphism $G \simeq \widehat{G}$ and we identify $G$ with $\widehat{\widehat{G}}$ via the natural isomorphism which takes $g \in G$ to the point evaluation map $(\chi \mapsto \chi(g)) \in \widehat{\widehat{G}}$. For a subgroup $K$ of $G$, let

$$
K^{\perp}:=\{\chi \in \widehat{G}: \chi(k)=1 \text { for all } k \in K\}
$$

which is a subgroup of $\widehat{G}$. With the above identification of $G$ and $\widehat{\widehat{G}}$, we have $K^{\perp \perp}:=\left(K^{\perp}\right)^{\perp}=K$.

We define $e: \mathbf{R} / \mathbf{Z} \rightarrow \mathbf{C}^{\times}$to be the injective group homomorphism $x+\mathbf{Z} \mapsto \exp (2 \pi i x)$. Since the additive group $\mathbf{R} / \mathbf{Z}$ embeds into the multiplicative group $\mathbf{C}^{\times}$of nonzero complex numbers via the map $x+$ $\mathbf{Z} \mapsto e(x)$, it follows that the additive group $H:=\operatorname{Hom}_{\mathbf{Z}}(G, \mathbf{R} / \mathbf{Z})$ is isomorphic to the multiplicative group $\widehat{G}$ via the map $\phi \mapsto e \circ \phi$. In this paper it will typically be more convenient to state results and proofs in terms of $H$ rather than $\widehat{G}$. However, we will sometimes take advantage of both the multiplicative and additive structure offered by C and work with $\widehat{G}$ instead.

\section{Overview of RESUlts}

We begin with our generalization of Reid's Terminal Lemma as claimed in the abstract. Let $\Lambda \subset \mathbf{R}^{n}$ be a lattice containing $\mathbf{Z}^{n}$. For $i=1,2, \ldots, n$, let $\pi_{i}: \Lambda / \mathbf{Z}^{n} \rightarrow \mathbf{R} / \mathbf{Z}$ denote the coordinate projection map sending $\left(\lambda_{1}, \ldots, \lambda_{n}\right)+\mathbf{Z}^{n}$ to $\lambda_{i}+\mathbf{Z}$. Observe that these maps are homomorphisms in the additive group $\operatorname{Hom}_{\mathbf{Z}}\left(\Lambda / \mathbf{Z}^{n}, \mathbf{R} / \mathbf{Z}\right)$ under pointwise addition; thus, it makes sense to talk about $-\pi_{i}$ for each $i$. By restricting to the appropriate subspace of $\mathbf{R}^{n}$, we assume without loss of generality that $\operatorname{ker} \pi_{i} \neq \Lambda / \mathbf{Z}^{n}$ for any $i$.

Theorem 3.1 (Terminal Lemma, c.f. [15, Theorem 5.4]). Let $u=$ $\left(u_{1}, \ldots, u_{n}\right) \in \mathbf{R}^{n}$. Then $\langle u, \lambda\rangle=0$ for every $\lambda \in \Lambda \cap[0,1)^{n}$ if and only if

$$
\sum_{\substack{i=1 \\ \pi_{i}=\pi_{j}}}^{n} u_{i}=\sum_{\substack{i=1 \\ \pi_{i}=-\pi_{j}}}^{n} u_{i}
$$

and

$$
\sum_{\substack{i=1 \\ \operatorname{ker} \pi_{i}=\operatorname{ker} \pi_{j}}}^{n} u_{i}=0
$$

for each $j=1,2, \ldots, n$. 
From this theorem several corollaries can be deduced. The first shows that the dimension of the span of the lattice points of $\Lambda$ in the half-open unit cube $[0,1)^{n}$ can be computed explicitly in terms of the coordinate projection functions $\pi_{i}: \Lambda / \mathbf{Z}^{n} \rightarrow \mathbf{R} / \mathbf{Z}$.

Let $\mathscr{I}$ denote the equivalence classes of the equivalence relation on the coordinates $\{1,2, \ldots, n\}$ in which $i \sim j$ in $\mathscr{I}$ if and only if $\pi_{i}=\pi_{j}$ or $\pi_{i}=-\pi_{j}$. Let $\mathscr{K}$ denote the equivalence classes of the equivalence relation on the coordinates $\{1,2, \ldots, n\}$ where $i \sim j$ in $\mathscr{K}$ if and only if $\operatorname{ker}\left(\pi_{i}\right)=\operatorname{ker}\left(\pi_{j}\right)$. Note that $\mathscr{K}$ coarsens $\mathscr{I}$ since $\operatorname{ker}\left(\pi_{i}\right)=\operatorname{ker}\left(-\pi_{i}\right)$ for all $i$.

Corollary 3.2. The dimension of $\operatorname{span}\left(\Lambda \cap[0,1)^{n}\right)$ is equal to $\iota+\kappa$, where $\iota$ denotes the number of equivalence classes $[i] \in \mathscr{I}$ such that $\pi_{i} \neq$ $-\pi_{i}$ and $\kappa$ denotes the number of equivalence classes in $\mathscr{K}$ containing coordinates $i, j$ (possibly equal) in which $\pi_{i}=-\pi_{j}$.

Proof. The distinct relations of the form (3.1) are in 1-1 correspondence with the equivalence classes $[i] \in \mathscr{I}$ such that $\pi_{i} \neq-\pi_{i}$. Note that in case $\pi_{i}=-\pi_{i}$, the relation (3.1) is trivial. Similarly, the distinct relations of the form (3.2) are in 1-1 correspondence with the equivalence classes of $\mathscr{K}$. The collection of all these relations are linearly independent except in the case when some $J \in \mathscr{K}$ does not contain any coordinates $i, j$ for which $\pi_{i}=-\pi_{j}$. In this situation, the relation (3.2) corresponding to $J$ is already implied by the relations (3.1) corresponding to the equivalence classes $I \in \mathscr{I}$ contained in $J$. Thus, after excluding the relations corresponding to such $J \in \mathscr{K}$, we conclude that the space of $u \in \mathbf{R}^{n}$ in which $\langle u, \lambda\rangle=0$ for all $\lambda \in \Lambda \cap[0,1)^{n}$ has dimension $n-\iota-\kappa$ and hence the dimension of the span of $\Lambda \cap[0,1)^{n}$ equals $\iota+\kappa$.

Observe that it is always true that

$$
\lambda_{1}+\cdots+\lambda_{n}+\mu_{1}+\cdots+\mu_{n}=|\operatorname{supp}(\lambda)|=|\operatorname{supp}(\mu)|
$$

for every pair $\lambda, \mu \in \Lambda \cap[0,1)^{n}$ for which $\lambda+\mathbf{Z}^{n}=-\mu+\mathbf{Z}^{n}$. This follows from the fact that for every $i=1,2, \ldots, n$, we have either $\lambda_{i}=1-\mu_{i}$ if both $\lambda_{i}$ and $\mu_{i}$ are nonzero, or $\lambda_{i}=\mu_{i}=0$ otherwise. The next corollary characterizes the situation where the "mass" of $\lambda+\mu$ is distributed as equally as possible between $\lambda$ and $\mu$ for all such pairs $\lambda, \mu$. It is a direct generalization of Sebő's Conjecture 1.1.

Corollary 3.3 (c.f. [17, Conjecture 4.1], [2, Proposition 1.8]). The equality

$$
\lambda_{1}+\cdots+\lambda_{n}=\frac{|\operatorname{supp}(\lambda)|}{2}
$$


TABLE 1. An illustration of Theorem 3.1 and Corollary 3.2 for the lattice $\Lambda$ generated by $\mathbf{Z}^{8}$ and the two points $\lambda=\frac{1}{10}(1,9,3,7,1,1,3,5)$ and $\lambda^{\prime}=\frac{1}{10}(2,8,6,4,1,1,3,0)$. The coordinate projection maps $\pi_{i}: \Lambda / \mathbf{Z}^{8} \rightarrow \mathbf{R} / \mathbf{Z}$ are uniquely determined by the two numbers $\lambda_{i}$ and $\lambda_{i}^{\prime}$. In this example, $\iota=4$ (corresponding to the classes $I_{1}, I_{2}, I_{3}, I_{4}$ in $\mathscr{I}$ ) and $\kappa=2$ (corresponding to the classes $J_{1}, J_{3}$ in $\left.\mathscr{K}\right)$. By Corollary 3.2 , the dimension of the linear span of $\Lambda \cap[0,1)^{8}$ is 6 .

\begin{tabular}{|c|c|c|c|c|c|c|c|c|c|c|c|}
\hline $\mathscr{K}$ & \multicolumn{4}{|c|}{$J_{1}$} & & \multicolumn{3}{|c|}{$J_{2}$} & & \multirow{2}{*}{$\begin{array}{l}J_{3} \\
I_{5}\end{array}$} & \\
\hline $\mathscr{I}$ & \multicolumn{2}{|c|}{$I_{1}$} & \multicolumn{2}{|c|}{$I_{2}$} & & \multicolumn{2}{|c|}{$I_{3}$} & \multirow{2}{*}{$\begin{array}{c}I_{4} \\
7\end{array}$} & & & \\
\hline$i$ & 1 & 2 & 3 & 4 & & 5 & 6 & & & 8 & \\
\hline$\lambda_{i}$ & 0.1 & 0.9 & 0.3 & 0.7 & & 0.1 & 0.1 & 0.3 & & 0.5 & \\
\hline$\lambda_{i}^{\prime}$ & 0.2 & 0.8 & 0.6 & 0.4 & & 0.1 & 0.1 & 0.3 & & 0.0 & \\
\hline \multirow[t]{2}{*}{ (3.1) relations } & \multirow[t]{2}{*}{$u_{1}$} & \multirow[t]{2}{*}{$-u_{2}$} & & & $=0$ & \multirow[t]{2}{*}{$u_{5}$} & \multirow[t]{2}{*}{$+u_{6}$} & & $=0$ & \multirow[t]{2}{*}{$u_{8}-u_{8}$} & \multirow[t]{2}{*}{$=0$} \\
\hline & & & $u_{3}$ & $-u_{4}$ & $=0$ & & & $u_{7}$ & $=0$ & & \\
\hline (3.2) relations & $u_{1}$ & $+u_{2}$ & $+u_{3}$ & $+u_{4}$ & $=0$ & $u_{5}$ & $+u_{6}$ & $+u_{7}$ & $=0$ & $u_{8}$ & $=0$ \\
\hline
\end{tabular}

holds for all $\lambda=\left(\lambda_{1}, \ldots, \lambda_{n}\right) \in \Lambda \cap[0,1)^{n}$ if and only if there exists an involution $\sigma$ of $\{1,2, \ldots, n\}$ (i.e. a bijection satisfying $\sigma=\sigma^{-1}$ ) such that $\lambda_{i}+\lambda_{\sigma(i)}$ is an integer for all $i=1,2, \ldots, n$ and $\lambda \in \Lambda$.

Example 3.4. If the coordinates of the points in $\Lambda$ are all half-integral (i.e. $\Lambda \subset \frac{1}{2} \mathbf{Z}^{n}$ ), then Corollary 3.3 is trivial. Indeed, both the hypothesis and the conclusion always hold; for the conclusion we may take $\sigma$ to be the identity map.

Example 3.5. If $\Lambda$ is generated by $\mathbf{Z}^{n}$ and the point $\frac{1}{r}\left(a_{1}, a_{2}, \ldots, a_{n}\right)$, where the $a_{i}$ 's are positive integers coprime to $r$, then we recover Conjecture 1.1. Indeed, in this case $|\operatorname{supp}(\lambda)|=n$ for every nonzero $\lambda \in[0,1)^{n} \cap \Lambda$, and $\lambda_{i}+\lambda_{\sigma(i)} \in \mathbf{Z}$ for all $i=1,2, \ldots, n$ and $\lambda \in \Lambda$ if and only if $a_{i}+a_{\sigma(i)}=r$ for all $i=1,2, \ldots, n$. This follows from the fact that for every nonzero $\lambda \in \Lambda \cap[0,1)^{n}$ there exists $1 \leq k \leq r-1$ such that $\lambda_{i}=\left\{k a_{i} / r\right\} \neq 0$ for each $i=1,2, \ldots, n$.

Example 3.6. If $\Delta \subseteq \mathbf{R}^{n-1}$ is a lattice polytope, then the Ehrhart series of $\Delta$ is given by

$$
\operatorname{Ehr}_{\Delta}(t)=\sum_{m \geq 0}\left|m \Delta \cap \mathbf{Z}^{n-1}\right| t^{m}=\frac{1+h_{1}^{*} t+\cdots+h_{n-1}^{*} t^{n-1}}{(1-t)^{n}}
$$

and the numerator of the left-hand side is called the $h^{*}$-polynomial of $\Delta$. If furthermore $\Delta=\operatorname{conv}\left(v_{1}, \ldots, v_{n}\right)$ is a simplex, where each $v_{i} \in \mathbf{Z}^{n-1}$, 
then it is known that $h_{k}^{*}$ equals the number of $\lambda \in \Lambda_{\Delta} \cap[0,1)^{n}$ such that $\lambda_{1}+\cdots+\lambda_{n}=k$ [4, Corollary 3.11]. Here $\Lambda_{\Delta} \subset \mathbf{R}^{n}$ denotes the dual lattice of the lattice generated by $\left(v_{i}, 1\right) \in \mathbf{Z}^{n}$ for $i=1,2, \ldots, n$; equivalently, the lattice of points $\lambda \in \mathbf{R}^{n}$ such that $\left\langle\lambda,\left(v_{i}, 1\right)\right\rangle \in \mathbf{Z}$ for all $i$.

Polytopes $\Delta$ with $h^{*}$-polynomial of the form $1+h_{k}^{*} t^{k}$ for some positive $k$ have been completely classified by Batyrev and Hofscheier [3]. They show that such a polytope $\Delta$ must be a simplex; therefore, the corresponding lattice $\Lambda_{\Delta}$ has the property that $\lambda_{1}+\cdots+\lambda_{n}=k$ for all nonzero $\lambda \in \Lambda_{\Delta} \cap[0,1)^{n}$. It follows that the hypothesis of Corollary 3.3 applies to $\Lambda_{\Delta}$, and the resulting involution $\sigma$ appears in their classification. They also describe some properties that $\Lambda_{\Delta} / \mathbf{Z}^{n}$ has; for instance, $\Lambda_{\Delta} / \mathbf{Z}^{n}$ is isomorphic to the additive group of $\mathbf{F}_{p}^{r}$ for some prime $p$ and integer $r$, and the integer $k$ satisfies $\left(p^{r}-p^{r-1}\right) n=2 k\left(p^{r}-1\right)$.

Proof of Corollary 3.3. The "if" direction is an immediate consequence of the fact that, for every $x \in \mathbf{R},\{x\}+\{-x\}$ equals 1 if $x \notin \mathbf{Z}$ and 0 otherwise.

For the "only if" direction, consider the lattice $\Lambda^{\prime} \subset \mathbf{R}^{2 n}$ which is generated by $\mathbf{Z}^{2 n}$ and the image of the map $\Lambda \rightarrow \mathbf{R}^{2 n}$ defined by

$$
\left(\lambda_{1}, \ldots, \lambda_{n}\right) \mapsto\left(\lambda_{1}, \ldots, \lambda_{n},-\lambda_{1}, \ldots,-\lambda_{n}\right) .
$$

Let $\lambda \in \Lambda \cap[0,1)^{n}$ and let $\lambda^{\prime} \in \Lambda^{\prime} \cap[0,1)^{2 n}$ be the unique integral translate in $[0,1)^{2 n}$ of the image of $\lambda$ under this map. Let $\mu \in \Lambda \cap[0,1)^{n}$ the the unique lattice point in $[0,1)^{n}$ which satisfies $\lambda+\mathbf{Z}^{n}=-\mu+\mathbf{Z}^{n}$. Then

$$
\lambda_{1}^{\prime}+\cdots+\lambda_{n}^{\prime}=\lambda_{n+1}^{\prime}+\cdots+\lambda_{2 n}^{\prime}
$$

since by assumption we have

$$
\lambda_{1}^{\prime}+\cdots+\lambda_{n}^{\prime}=\lambda_{1}+\cdots+\lambda_{n}=\frac{|\operatorname{supp}(\lambda)|}{2}
$$

and

$$
\lambda_{n+1}^{\prime}+\cdots+\lambda_{2 n}^{\prime}=\mu_{1}+\cdots+\mu_{n}=\frac{|\operatorname{supp}(\mu)|}{2}
$$

and we know by the preceding discussion that $|\operatorname{supp}(\mu)|=|\operatorname{supp}(\lambda)|$. If we let

$$
u^{\prime}=(\underbrace{1, \ldots, 1}_{n}, \underbrace{-1, \ldots,-1}_{n}) \in \mathbf{R}^{2 n}
$$


we get $\left\langle u^{\prime}, \lambda^{\prime}\right\rangle=0$ for each $\lambda^{\prime} \in \Lambda^{\prime} \cap[0,1)^{2 n}$. We may therefore apply Theorem 3.1 to obtain the equality

$$
\left(\sum_{\substack{i=1 \\ \pi_{i}=\pi_{j}}}^{n} 1\right)-\left(\sum_{\substack{i=1 \\ \pi_{i}=-\pi_{j}}}^{n} 1\right)=\left(\sum_{\substack{i=1 \\ \pi_{i}=-\pi_{j}}}^{n} 1\right)-\left(\sum_{\substack{i=1 \\ \pi_{i}=\pi_{j}}}^{n} 1\right)
$$

for each $j \in\{1,2, \ldots, n\}$, which simplifies to

$$
\left|\left\{i: \pi_{i}=\pi_{j}\right\}\right|=\left|\left\{i: \pi_{i}=-\pi_{j}\right\}\right| .
$$

We now construct our involution $\sigma:\{1,2, \ldots, n\} \rightarrow\{1,2, \ldots, n\}$. For each $i$ such that $\pi_{i}=-\pi_{i}$, we set $\sigma(i)=i$. For each coordinate projection map $\pi$ such that $\pi \neq-\pi$, we pair up each coordinate $i$ such that $\pi_{i}=\pi$ with a unique coordinate $j$ such that $\pi_{j}=-\pi$. Then, for each such pair $(i, j)$, we set $\sigma(i)=j$ and $\sigma(j)=i$. Now let $\lambda \in \Lambda$ and let $i \in\{1,2, \ldots, n\}$. Then

$$
\lambda_{i}+\lambda_{\sigma(i)}+\mathbf{Z}=\pi_{i}(\lambda)+\left(-\pi_{i}\right)(\lambda)=0+\mathbf{Z}
$$

and hence $\lambda_{i}+\lambda_{\sigma(i)}$ is an integer.

The proof of Theorem [3.1, specifically the "only-if" direction, depends on the following two claims. The first is used to establish the relations (3.2) assuming the hypotheses of Theorem 3.1. The proof given in the next section relies on the Poisson summation formula for finite abelian groups.

Lemma 3.7. Let $G$ be a finite abelian group. For a subgroup $K$ of $G$, let $\mathbf{1}_{K} \in L^{2}(G)$ denote the indicator function of $K$. Then

$$
\left\{\mathbf{1}_{K}: K^{\perp} \text { is a cyclic subgroup of } \widehat{G}\right\}
$$

is linearly independent in $L^{2}(G)$.

We remark that this statement is quite easy to prove in the case when $G$ is cyclic.

The second claim is used to establish the relations (3.1) assuming the hypotheses of Theorem 3.1. Suppose $G$ is a finite abelian group and let $H=\operatorname{Hom}_{\mathbf{Z}}(G, \mathbf{R} / \mathbf{Z})$. Consider the space $L_{\text {odd }}^{2}(H)$ consisting of functions $f: H \rightarrow \mathbf{C}$ which satisfy $f(-\phi)=-f(\phi)$ for all $\phi \in H$. For each $g \in G$, define the function $S_{g} \in L^{2}(H)$ by

$$
S_{g}(\phi)=B_{1}(\phi(g)) \quad \text { for all } \phi \in H .
$$

Note that these functions lie in $L_{\text {odd }}^{2}(H)$ since $B_{1}$ is an odd function. Crucially, however, much more is true:

Theorem 3.8 (c.f. [13, Proposition 1.2]). The space $L_{\text {odd }}^{2}(H)$ is spanned by the functions $S_{g}$ for $g \in G$. 
We remark that these functions are closely related to the Stickelberger distribution associated with $B_{1}$ described in [10, Chapter 2]. As in [13, 15, the proof of this theorem relies on Dirichlet's theorem that $L(1, \chi) \neq 0$ for a nontrivial Dirichlet character $\chi$ where $L(s, \chi)$ denotes the Dirichlet $L$-function associated with $\chi$.

\section{Proof of Theorem 3.1}

We make some preliminary observations before stating the proof. Let $H=\operatorname{Hom}_{\mathbf{Z}}\left(\Lambda / \mathbf{Z}^{n}, \mathbf{R} / \mathbf{Z}\right)$. Given $u=\left(u_{1}, \ldots, u_{n}\right) \in \mathbf{R}^{n}$, define $h_{u} \in L^{2}(H)$ to be the function

$$
h_{u}(\phi)=\left(\sum_{\substack{i=1 \\ \pi_{i}=\phi}}^{n} u_{i}\right)-\left(\sum_{\substack{i=1 \\ \pi_{i}=-\phi}}^{n} u_{i}\right) \quad \text { for all } \phi \in H .
$$

Also define as above, for each $\lambda \in \Lambda \cap[0,1)^{n}$, the function $S_{\lambda}: H \rightarrow \mathbf{C}$ :

$$
S_{\lambda}(\phi)=B_{1}\left(\phi\left(\lambda+\mathbf{Z}^{n}\right)\right)= \begin{cases}\left\{\phi\left(\lambda+\mathbf{Z}^{n}\right)\right\}-1 / 2, & \phi\left(\lambda+\mathbf{Z}^{n}\right) \neq 0+\mathbf{Z} \\ 0, & \phi\left(\lambda+\mathbf{Z}^{n}\right)=0+\mathbf{Z}\end{cases}
$$

The most important property about these functions is that they are odd functions; we have $S_{\lambda}(-\phi)=-S_{\lambda}(\phi)$ for each $\phi \in H$ and $\lambda \in \Lambda \cap[0,1)^{n}$.

Observe that for any $\lambda \in \Lambda \cap[0,1)^{n}$, we have

$$
\sum_{i=1}^{n} u_{i} \lambda_{i}=\left(\sum_{i=1}^{n} u_{i} S_{\lambda}\left(\pi_{i}\right)\right)+\frac{1}{2}\left(\sum_{\substack{i=1 \\ \lambda_{i} \neq 0}}^{n} u_{i}\right)
$$

Since $\phi \mapsto-\phi$ is a permutation of $H$, we may write the first term as

$$
\begin{aligned}
\sum_{\phi \in H} \sum_{\substack{i=1 \\
\pi_{i}=\phi}}^{n} u_{i} S_{\lambda}(\phi) & =\frac{1}{2} \sum_{\phi \in H}\left(\left(\sum_{\substack{i=1 \\
\pi_{i}=\phi}}^{n} u_{i} S_{\lambda}(\phi)\right)+\left(\sum_{\substack{i=1 \\
\pi_{i}=-\phi}}^{n} u_{i} S_{\lambda}(-\phi)\right)\right) \\
& =\frac{1}{2} \sum_{\phi \in H}\left(\left(\sum_{\substack{i=1 \\
\pi_{i}=\phi}}^{n} u_{i}\right)-\left(\sum_{\substack{i=1 \\
\pi_{i}=-\phi}}^{n} u_{i}\right)\right) S_{\lambda}(\phi) \\
& =\frac{|H|}{2}\left\langle h_{u}, S_{\lambda}\right\rangle
\end{aligned}
$$

where the second-to-last equality follows from the fact that $S_{\lambda}$ is an

odd function. So we conclude that for any $u \in \mathbf{R}^{n}$ with corresponding 
$h_{u} \in L^{2}(H)$ as defined above, and for any $\lambda \in \Lambda \cap[0,1)^{n}$, we have

$$
\sum_{i=1}^{n} u_{i} \lambda_{i}=\frac{1}{2}\left(|H|\left\langle h_{u}, S_{\lambda}\right\rangle+\sum_{\substack{i=1 \\ \lambda_{i} \neq 0}}^{n} u_{i}\right)
$$

Proof of the if direction of Theorem 3.1. We start with the easier direction. Assume $u \in \mathbf{R}^{n}$ satisfy the relations (3.1) and (3.2) and let $\lambda \in \Lambda \cap[0,1)^{n}$. The relations (3.1) imply that $h_{u}$ is the zero function, so by (4.1) we may therefore write

$$
\sum_{i=1}^{n} u_{i} \lambda_{i}=\frac{1}{2} \sum_{\substack{i=1 \\ \lambda_{i} \neq 0}}^{n} u_{i}=\frac{1}{2} \sum_{K} \sum_{\substack{i=1 \\ \lambda i \neq 0 \\ \operatorname{ker} \pi_{i}=K}}^{n} u_{i}=\frac{1}{2} \sum_{\substack{K \\ \lambda+\mathbf{Z}^{n} \notin K}} \sum_{\substack{i=1 \\ \operatorname{ker} \pi_{i}=K}}^{n} u_{i}
$$

where the outer sums are over all subgroups $K \in\left\{\operatorname{ker} \pi_{i}: i=1,2, \ldots, n\right\}$. By (3.2), the inner sums of the double sum on the right always vanish, and therefore the whole expression equals zero.

Proof of the only if direction. Let $u \in \mathbf{R}^{n}$ with corresponding $h_{u} \in$ $L^{2}(H)$ as defined above, and assume that $\langle u, \lambda\rangle=0$ for every $\lambda \in$ $\Lambda \cap[0,1)^{n}$. For every pair $\lambda, \mu \in \Lambda \cap[0,1)^{n}$ such that $\lambda+\mathbf{Z}^{n}=-\mu+\mathbf{Z}^{n}$, we have

$$
\sum_{\substack{i=1 \\ \lambda_{i} \neq 0}}^{n} u_{i}=\left(\sum_{i=1}^{n} u_{i} \lambda_{i}\right)+\left(\sum_{i=1}^{n} u_{i} \mu_{i}\right)=0
$$

by our assumption that both the terms in the middle vanish. Hence

$$
\frac{|H|}{2}\left\langle h_{u}, S_{\lambda}\right\rangle=\sum_{i=1}^{n} u_{i} \lambda_{i}=0
$$

for every $\lambda \in \Lambda \cap[0,1)^{n}$ by equation (4.1). So by Theorem [3.8, $h_{u}$ is orthogonal to every odd function in $L^{2}(H)$ and therefore must be an even function. But $h_{u}$ is an odd function by definition. It follows $h_{u}$ must be the zero map, and therefore the relations (3.1) hold.

We next show that the relations (3.2) hold as well. From (4.2), we have

$$
\left(\sum_{i=1}^{n} u_{i}\right)-\sum_{\substack{i=1 \\ \lambda_{i}=0}}^{n} u_{i}=0
$$

for all $\lambda \in \Lambda \cap[0,1)^{n}$ which implies

$$
\left(\sum_{i=1}^{n} u_{i}\right) \mathbf{1}_{\Lambda / \mathbf{Z}^{n}}-\sum_{i=1}^{n} u_{i} \mathbf{1}_{\mathrm{ker} \pi_{i}}=\mathbf{0}
$$


where $\mathbf{1}_{K} \in L^{2}\left(\Lambda / \mathbf{Z}^{n}\right)$ denotes the indicator function of the subgroup $K$ of $\Lambda / \mathbf{Z}^{n}$ and $\mathbf{0}$ denotes the zero map. We may rewrite this sum as

$$
\left(\sum_{i=1}^{n} u_{i}\right) \mathbf{1}_{\Lambda / \mathbf{Z}^{n}}-\sum_{K}\left(\sum_{\substack{i=1 \\ \operatorname{ker} \pi_{i}=K}}^{n} u_{i}\right) \mathbf{1}_{K}=\mathbf{0}
$$

where the second sum is over all subgroups $K \in\left\{\operatorname{ker} \pi_{i}: i=1,2, \ldots, n\right\}$. Let $e: \mathbf{R} / \mathbf{Z} \rightarrow \mathbf{C}^{\times}$be the map $x+\mathbf{Z} \mapsto \exp (2 \pi i x)$. Then $e \circ \pi_{i} \in$ $\widehat{\Lambda / \mathbf{Z}^{n}}$ and, moreover, $\operatorname{ker} \pi_{i}=\left\langle e \circ \pi_{i}\right\rangle^{\perp}$ for each $i=1,2, \ldots, n$. We also have $\Lambda / \mathbf{Z}^{n}=\left\langle\chi_{0}\right\rangle^{\perp}$, where $\chi_{0}$ denotes the identity of $\widehat{\Lambda / \mathbf{Z}^{n}}$. It follows that $\left(\operatorname{ker} \pi_{i}\right)^{\perp}$ for $i=1,2, \ldots, n$ and $\left(\Lambda / \mathbf{Z}^{n}\right)^{\perp}$ are all cyclic subgroups of $\widehat{\Lambda / \mathbf{Z}^{n}}$. By Lemma 3.7, then, the set of indicator functions in the above linear combination are linearly independent. We conclude each of the coefficients of the indicator functions above are zero, and therefore the relations (3.2) hold. Note that there is no $\mathbf{1}_{\Lambda / \mathbf{Z}^{n}}$ term among the sum of $\mathbf{1}_{K}$ 's due to the assumption that ker $\pi_{i} \neq \Lambda / \mathbf{Z}^{n}$ for every $i$.

\section{Proof of Lemma 3.7}

Let $G$ be a finite abelian group. If $f \in L^{2}(G)$, we define the Fourier transform $\hat{f} \in L^{2}(\widehat{G})$ by

$$
\hat{f}(\chi)=\langle f, \chi\rangle=\frac{1}{|G|} \sum_{g \in G} f(g) \overline{\chi(g)}
$$

for every $\chi \in \widehat{G}$. Since the characters of $G$ form an orthonormal basis of $L^{2}(G)$, we have in particular that

$$
\widehat{\psi}(\chi)=\langle\psi, \chi\rangle= \begin{cases}1, & \psi=\chi \\ 0, & \psi \neq \chi\end{cases}
$$

for every $\psi, \chi \in \widehat{G}$.

Lemma 3.7 is essentially a consequence of the Poisson summation formula for finite abelian groups, stated below. We refer the reader to [7, Exercise 4.6] or [18, Chapter 12] for references.

Proposition 5.1 (Poisson summation formula). Let $G$ be a finite abelian group, let $f \in L^{2}(G)$, and let $K$ be a subgroup of $G$. Then

$$
\frac{1}{|G|} \sum_{k \in K} f(k)=\frac{1}{\left|K^{\perp}\right|} \sum_{\chi \in K^{\perp}} \hat{f}(\chi) .
$$

Lemma 5.2. Let $\mathcal{K}$ be a collection of subgroups of $G$ with the property that $\left\{\mathbf{1}_{K^{\perp}}: K \in \mathcal{K}\right\}$ is linearly independent in $L^{2}(\widehat{G})$. Then $\left\{\mathbf{1}_{K}: K \in \mathcal{K}\right\}$ is linearly independent in $L^{2}(G)$. 
Proof. Let $\mathcal{K}$ be such a collection, and suppose

$$
\sum_{K \in \mathcal{K}} \alpha_{K} \mathbf{1}_{K}=0
$$

for some complex numbers $\alpha_{K}$ for $K \in \mathcal{K}$. Thus for any character $\psi \in \widehat{G}$, we obtain

$$
\sum_{K \in \mathcal{K}} \frac{\alpha_{K}}{\left|K^{\perp}\right|} \sum_{\chi \in K^{\perp}} \widehat{\psi}(\chi)=\sum_{K \in \mathcal{K}} \frac{\alpha_{K}}{|G|} \sum_{k \in K} \psi(k)=\left\langle\sum_{K \in \mathcal{K}} \alpha_{K} \mathbf{1}_{K}, \bar{\psi}\right\rangle=0
$$

by the Poisson summation formula. On the other hand, by (5.1), the left hand side simplifies to

$$
\sum_{\substack{K \in \mathcal{K} \\ \psi \in K^{\perp}}} \frac{\alpha_{K}}{\left|K^{\perp}\right|}=\sum_{K \in \mathcal{K}} \frac{\alpha_{K}}{\left|K^{\perp}\right|} \mathbf{1}_{K^{\perp}}(\psi) .
$$

It follows that the linear combination of functions

$$
\sum_{K \in \mathcal{K}} \frac{\alpha_{K}}{\left|K^{\perp}\right|} \mathbf{1}_{K^{\perp}} \in L^{2}(\widehat{G})
$$

is the zero function. Since the functions $\left\{\mathbf{1}_{K^{\perp}}: K \in \mathcal{K}\right\}$ are assumed to be linearly independent, we get that each $\alpha_{K}=0$ which is what we wanted to show.

Recall Lemma 3.7, which claims $\left\{\mathbf{1}_{K}: K^{\perp}\right.$ is a cyclic subgroup of $\left.\widehat{G}\right\}$ is linearly independent in $L^{2}(G)$.

Proof of Lemma 3.7. Let $\mathcal{K}=\left\{\langle\chi\rangle^{\perp}: \chi \in \widehat{G}\right\}$. By the preceding lemma, it suffices to show that set of functions

$$
\left\{\mathbf{1}_{K^{\perp}}: K \in \mathcal{K}\right\}=\left\{\mathbf{1}_{\langle\chi\rangle}: \chi \in \widehat{G}\right\}
$$

is linearly independent in $L^{2}(\widehat{G})$.

The cyclic subgroups of $\widehat{G}$ form a partially ordered set with respect to inclusion. Hence, by taking any linear extension of this poset, we enumerate these subgroups as $\left\langle\chi_{1}\right\rangle,\left\langle\chi_{2}\right\rangle, \ldots,\left\langle\chi_{n}\right\rangle$ in such a way that $i<j$ implies there is an element of $\left\langle\chi_{j}\right\rangle$ not in $\left\langle\chi_{i}\right\rangle$. This implies that the matrix

$$
A_{i, j}= \begin{cases}1, & \chi_{i} \in\left\langle\chi_{j}\right\rangle \\ 0, & \text { otherwise }\end{cases}
$$

where $1 \leq i, j \leq n$, is upper triangular with ones along the diagonal. It follows that the functions $\mathbf{1}_{\langle\chi\rangle}: \chi \in \widehat{G}$ are linearly independent, as they are linearly independent when restricted to $\left\{\chi_{1}, \ldots, \chi_{n}\right\}$. 


\section{Proof of Theorem 3.8}

Let $G$ be a finite abelian group written additively, and let $H=$ $\operatorname{Hom}(G, \mathbf{R} / \mathbf{Z})$. We wish to show that the space $L_{\text {odd }}^{2}(H)$ of odd functions $f: H \rightarrow \mathbf{C}$ is spanned by the functions $S_{g}: H \rightarrow \mathbf{C}$ defined by $S_{g}(\phi)=B_{1}(\phi(g))$ for each $g \in G$. The proof of this statement is outlined in this section, and follows the methods of [15, 13] by explicitly finding $\operatorname{dim}\left(L_{\text {odd }}^{2}(H)\right)$ many linearly independent vectors in $\operatorname{span}\left(S_{g}: g \in G\right)$.

6.1. Some preliminaries. By the structure theorem for finitely generated abelian groups, $G \simeq H$ is isomorphic to an additive group of the form

$$
\bigoplus_{i=1}^{m} \mathbf{Z} / r_{i}
$$

where $r_{1}, r_{2}, \ldots, r_{m}$ are positive integers such that $m \geq 1$ and $r_{1}\left|r_{2}\right|$ $\cdots \mid r_{m}$. Now fix a minimal set of generators $\left\{g_{1}, \ldots, g_{m}\right\}$ of $G$, so that every element $g \in G$ can be written uniquely as $a_{1} g_{1}+\cdots+a_{m} g_{m}$ for some integers $a_{1}, \ldots, a_{m}$ satisfying $0 \leq a_{i}<r_{i}$ for $i=1,2, \ldots, m$. Then the maps $\phi_{i} \in H$ for $i=1,2, \ldots, m$ defined by

$$
\phi_{i}\left(g_{j}\right)= \begin{cases}0+\mathbf{Z} & i \neq j \\ \frac{1}{r_{i}}+\mathbf{Z} & i=j\end{cases}
$$

are a minimal generating set for $H$ in that every $\phi \in H$ can be written uniquely as $c_{1} \phi_{1}+\cdots+c_{m} \phi_{m}$ for some integers $c_{1}, \ldots, c_{m}$ satisfying $0 \leq c_{i}<r_{i}$ for $i=1,2, \ldots, m$. Moreover, given $g \in G$ and $\phi \in H$, if $g=a_{1} g_{1}+\cdots+a_{m} g_{m}$ and $\phi=c_{1} \phi_{1}+\cdots+c_{m} \phi_{m}$, then

$$
\phi(g)=\frac{a_{1} c_{1}}{r_{1}}+\cdots+\frac{a_{m} c_{m}}{r_{m}}+\mathbf{Z} .
$$

Now let $R$ denote the $\operatorname{ring} \mathbf{Z} / r_{1} \oplus \cdots \oplus \mathbf{Z} / r_{m}$ with componentwise multiplication, so that the additive group of $R$ is isomorphic to $G$. For each $a=\left(a_{1}, \ldots, a_{m}\right) \in R$, define the function $S_{a}: R \rightarrow \mathbf{C}$ by

$$
S_{a}(c)=B_{1}\left(\frac{a_{1} c_{1}}{r_{1}}+\cdots+\frac{a_{m} c_{m}}{r_{m}}\right)
$$

for each $c=\left(c_{1}, \ldots, c_{m}\right) \in R$.

As before, let $L_{\text {odd }}^{2}(R)$ denote the space of functions $f: R \rightarrow \mathbf{C}$ satisfying $f(-a)=-f(a)$ for all $a \in R$. Theorem 3.8, then, is established by proving the following proposition:

Proposition 6.1. The functions in $\left\{S_{a}: a \in R\right\}$ span $L_{\text {odd }}^{2}(R)$. 
Before proceeding with the proof of Proposition 6.1, we review the notion of Dirichlet characters and establish the notation to be used in the remainder of this section. A reference can be found in [11, Section $9.1]$.

6.2. Dirichlet characters. Let $G=(\mathbf{Z} / r)^{\times}$for some positive integer $r$. Then each character $\chi: G \rightarrow \mathbf{C}^{\times}$extends to a completely multiplicative function $\chi: \mathbf{Z} \rightarrow \mathbf{C}$ by setting

$$
\chi(n):= \begin{cases}\chi(n+r \mathbf{Z}), & \operatorname{gcd}(n, r)=1 \\ 0, & \text { otherwise }\end{cases}
$$

for each integer $n$. A function $\chi: \mathbf{Z} \rightarrow \mathbf{C}$ is called a Dirichlet character if it is constructed in this manner for some $r \geq 1$ and some $\chi \in \widehat{(\mathbf{Z} / r)^{x}}$. The number $r$ is called the modulus of $\chi$. We define an equivalence relation $\sim$ on Dirichlet characters by declaring $\chi_{1} \sim \chi_{2}$ if and only if they agree on their mutual support. A Dirichlet character $\chi$ is called primitive if the support of $\chi$ contains the support of every other Dirichlet character in the equivalence class $[\chi]$. Given a Dirichlet character $\chi$, there exists a unique primitive Dirichlet character in the equivalence class $[\chi]$ and it is denoted $\chi^{*}$. A primitive character $\chi^{*}$ is said to induce a Dirichlet character $\psi$ if $\psi \in\left[\chi^{*}\right]$. If $\chi$ is a Dirichlet character, then the modulus of $\chi^{*}$ is called the conductor of $\chi$.

6.3. Notation. We outline the notation used in the remainder of this section.

\subsubsection{Arithmetic functions. Let $\mathbf{N}$ denote the positive integers.}

- $\nu_{p}: \mathbf{N} \rightarrow \mathbf{Z}$ denotes the $p$-adic valuation: $\nu_{p}(k)$ is the largest exponent $\alpha$ such that $p^{\alpha} \mid k$.

- $\mathrm{d}: \mathbf{N} \rightarrow \mathbf{N}$ counts the number of divisors of an integer: we have $\mathrm{d}(k)=\prod_{p}\left(\nu_{p}(k)+1\right)$ for all $k \geq 1$ where the product is over all primes $p$.

- $\mu: \mathbf{N} \rightarrow \mathbf{Z}$ is the Möbius function.

- $\varphi: \mathbf{N} \rightarrow \mathbf{N}$ is the Euler-phi function.

- We write $\left(k, k^{\prime}\right)$ for the greatest common divisor of $k$ and $k^{\prime}$.

For $a=\left(a_{1}, \ldots, a_{m}\right) \in \mathbf{N}^{m}$, we also define

- $\mathrm{d}(a):=\mathrm{d}\left(a_{1}\right) \mathrm{d}\left(a_{2}\right) \cdots \mathrm{d}\left(a_{m}\right)$.

- $\mu(a):=\mu\left(a_{1}\right) \mu\left(a_{2}\right) \cdots \mu\left(a_{m}\right)$.

- $\varphi(a):=\varphi\left(a_{1}\right) \varphi\left(a_{2}\right) \cdots \varphi\left(a_{m}\right)$. 
6.3.2. Dirichlet characters. Let $R=\oplus_{i=1}^{m} \mathbf{Z} / r_{i}$ be the ring defined above. The multiplicative group of units of $R$ is given by

$$
R^{\times}=\bigoplus_{i=1}^{m}\left(\mathbf{Z} / r_{i}\right)^{\times}
$$

Let $\widehat{R^{\times}}$denote the group of characters of $R^{\times}$. Each $\chi \in \widehat{R^{\times}}$corresponds uniquely to a tuple $\left(\chi_{1}, \ldots, \chi_{m}\right)$ for which $\left.\chi_{i} \in \overline{\left(\mathbf{Z} / r_{i}\right.}\right)^{\times}$for $i=1,2, \ldots, m$, and

$$
\chi(a)=\chi_{1}\left(a_{1}\right) \cdots \chi_{m}\left(a_{m}\right)
$$

for each $a=\left(a_{1}, \ldots, a_{m}\right) \in R^{\times}$. For a character $\chi_{i}:\left(\mathbf{Z} / r_{i}\right)^{\times} \rightarrow \mathbf{C}^{\times}$, we denote the corresponding Dirichlet character by $\chi_{i}: \mathbf{Z} \rightarrow \mathbf{C}$. For $\chi=\left(\chi_{1}, \ldots, \chi_{m}\right) \in \widehat{R^{\times}}$, we define

- $\chi: \mathbf{Z}^{m} \rightarrow \mathbf{C}$ by $\chi\left(a_{1}, \ldots, a_{m}\right)=\chi_{1}\left(a_{1}\right) \chi_{2}\left(a_{2}\right) \cdots \chi_{m}\left(a_{m}\right)$.

- $\chi^{*}: \mathbf{Z}^{m} \rightarrow \mathbf{C}$ by $\chi^{*}\left(a_{1}, \ldots, a_{m}\right)=\chi_{1}^{*}\left(a_{1}\right) \chi_{2}^{*}\left(a_{2}\right) \cdots \chi_{m}^{*}\left(a_{m}\right)$.

Here we are denoting by $\chi_{i}^{*}: \mathbf{Z} \rightarrow \mathbf{C}$ the primitive Dirichlet character inducing $\chi_{i}: \mathbf{Z} \rightarrow \mathbf{C}$.

6.3.3. Parameters associated with $R$. For the $\operatorname{ring} R$ defined above, and for each $\chi=\left(\chi_{1}, \ldots \chi_{m}\right) \in \widehat{R^{\times}}$, we define

- $r:=\left(r_{1}, \ldots, r_{m}\right)$

- $f_{\chi}:=\left(f_{\chi_{1}}, \ldots, f_{\chi_{m}}\right)$ where $f_{\chi_{i}}$ is the conductor of $\chi_{i}: \mathbf{Z} \rightarrow \mathbf{C}$.

- $q_{\chi}:=\left(r_{1} / f_{\chi_{1}}, \ldots, r_{m} / f_{\chi_{m}}\right)$.

6.3.4. Everything else. For two tuples of integers $a=\left(a_{1}, \ldots, a_{m}\right), c=$ $\left(c_{1}, \ldots, c_{m}\right) \in \mathbf{Z}^{m}$, we write $a c$ to denote the componentwise product $\left(a_{1} c_{1}, \ldots, a_{m} c_{m}\right)$. If $a$ and $c$ have positive components, then we write $a \mid c$ and say $a$ divides $c$ if $a_{i} \mid c_{i}$ for all $i=1,2, \ldots, m$. If $a$ divides $c$, then we let $c / a:=\left(c_{1} / a_{1}, \ldots, c_{m} / a_{m}\right)$. Thus, for instance, $q_{\chi}=r / f_{\chi}$ where $r, q_{\chi}, f_{\chi}$ are as above.

If $g, h: \mathbf{N}^{m} \rightarrow \mathbf{C}$, then let $*$ denote Dirichlet convolution over $\mathbf{N}^{m}$ :

$$
(g * h)(a):=\sum_{d \mid a} g(d) h(a / d) \quad \text { for all } a \in \mathbf{N}^{m} .
$$

6.4. A decomposition of $R$. The group of units $R^{\times}$of the $\operatorname{ring} R$ acts on $L^{2}(R)$ as follows: for a given $f \in L^{2}(R), c \in R^{\times}$, the function $c \cdot f \in L^{2}(R)$ is defined so that

$$
(c \cdot f)(a)=f(c a)
$$

for each $a \in R$. An eigenvalue, eigenfunction pair $(\chi, w)$ of the action consists of a function $\chi: R^{\times} \rightarrow \mathbf{C}$ and a nonzero function $w \in L^{2}(R)$ 
such that for every $c \in R^{\times}$,

$$
c \cdot w=\chi(c) w .
$$

The vector space $L^{2}(R)$ can be decomposed into a direct sum

$$
L^{2}(R)=\bigoplus_{\chi \in \widehat{R}^{x}} \varepsilon_{\chi}
$$

where, for each $\chi \in \widehat{R^{\times}}$, we denote the subspace of eigenfunctions corresponding to $\chi$ by $\varepsilon_{\chi}$.

Proposition 6.2. Let $\chi \in \widehat{R^{\times}}$. Then

$$
\varepsilon_{\chi}=\left\{\frac{1}{\left|R^{\times}\right|} \sum_{b \in R^{\times}} \bar{\chi}(b)(b \cdot w): w \in L^{2}(R)\right\} .
$$

Proof. If $w$ is an eigenfunction with eigenvalue $\chi$, then $w$ equals the average of $\bar{\chi}(b)(b \cdot w)$ over all $b \in R^{\times}$. Conversely, if $w \in L^{2}(R)$, then

$$
\begin{aligned}
c \cdot \sum_{b \in R^{\times}} \bar{\chi}(b)(b \cdot w)=\sum_{b \in R^{\times}} \bar{\chi}(b)(c b \cdot w) & =\chi(c) \sum_{b \in R^{\times}} \bar{\chi}(c b)(c b \cdot w) \\
& =\chi(c) \sum_{b \in R^{\times}} \bar{\chi}(b)(b \cdot w) .
\end{aligned}
$$

We say that a character $\chi \in \widehat{R^{\times}}$is even if $\chi(-1, \ldots,-1)=1$ and odd if $\chi(-1, \ldots,-1)=-1$; note that these are the only two possible values for $\chi(-1, \ldots,-1)$ since

$$
(\chi(-1, \ldots,-1))^{2}=\chi\left((-1, \ldots,-1)^{2}\right)=\chi(1, \ldots, 1)=1 .
$$

Observe that the functions in $\varepsilon_{\chi}$ are odd if and only if $\chi$ is odd.

For a given $\chi \in \widehat{R^{\times}}$and $a^{\prime} \in R$, let

$$
w_{\chi, a^{\prime}}:=\sum_{b \in R^{\times}} \chi(b) S_{a^{\prime} b} \in \varepsilon_{\chi} .
$$

If $a \in \mathbf{Z}^{m}$ and $a^{\prime} \in R$ is the image of $a$ under the canonical map $\mathbf{Z}^{m} \rightarrow R$, then we also define $w_{\chi, a}:=w_{\chi, a^{\prime}}$.

The next theorem, proved by Reid in [15] for the case when $m=1$, finds a basis of $\varepsilon_{\chi}$ in terms of these $w_{\chi, a}$ when $\chi$ is odd.

Proposition 6.3 (c.f. [15, Theorem 5.13]). For each odd character $\chi \in \widehat{R^{\times}}$, there are $\mathrm{d}\left(q_{\chi}\right)$ functions in $\left\{w_{\chi, a}: a \in \mathbf{N}^{m}, a \mid q_{\chi}\right\}$ and they are linearly independent.

With this proposition, we can prove Proposition 6.1 and hence Theorem 3.8 , 
ON THE LINEAR SPAN OF LATTICE POINTS IN A PARALLELEPIPED 18

Proof of Proposition [6.1. For a tuple $f=\left(f_{1}, \ldots, f_{m}\right) \in \mathbf{N}^{m}$, let $\hat{\varphi}_{\text {odd }}(f)$ denote the number of odd characters $\chi \in \widehat{R^{\times}}$such that $f=f_{\chi}$. Using Proposition 6.3 and the decomposition (6.1), we take the union of the sets $\left\{w_{\chi, a}: a \mid q_{\chi}\right\}$ over all odd characters $\chi$ to obtain $\left(\hat{\varphi}_{\text {odd }} * \mathrm{~d}\right)(r)$ linearly independent functions in $L_{\text {odd }}^{2}(R)$. We would therefore like to show that this number is equal to $\operatorname{dim}\left(L_{\text {odd }}^{2}(R)\right)$.

Since $*$ is associative, we get

$$
\left(\hat{\varphi}_{\text {odd }} * \mathrm{~d}\right)(r)=\left(\hat{\varphi}_{\text {odd }} * 1 * 1\right)(r)=\sum_{f \mid r}\left(\hat{\varphi}_{\text {odd }} * 1\right)(f) .
$$

Now each term $\left(\hat{\varphi}_{\text {odd }} * 1\right)(f)$ in the sum is equal to the total number of odd characters of the group $G_{f}:=\oplus_{i=1}^{m}\left(\mathbf{Z} / f_{i}\right)^{\times}$. This number is equal to zero if $G_{f}$ is the trivial group, which is the case if and only if $f_{i}$ equals one or two for every $i=1,2, \ldots, m$. Otherwise, $\left\{\psi \in \widehat{G_{f}}\right.$ : $\psi(-1, \ldots,-1)=1\}$ is an order two subgroup of $\widehat{G_{f}}$ and hence there are $\frac{1}{2}\left|\widehat{G_{f}}\right|=\frac{1}{2}\left|G_{f}\right|=\frac{1}{2} \varphi(f)$ odd characters in $\widehat{G_{f}}$.

If we let $\delta(f)=1$ whenever every component of $f$ is either 1 or 2 and zero otherwise, then we obtain

$$
\left(\hat{\varphi}_{\text {odd }} * d\right)(r)=\frac{1}{2} \sum_{f \mid r}(\varphi(f)-\delta(f))=\frac{1}{2}\left(r_{1} \cdots r_{m}-2^{s}\right)
$$

where $s$ equals the number of $i \in\{1,2, \ldots, m\}$ such that $r_{i}$ is even. Hence we obtain that the dimension of $\operatorname{span}\left(S_{a}: a \in R\right)$ is at least $\frac{1}{2}\left(|R|-2^{s}\right)$.

It remains to show that $\operatorname{dim}\left(L_{\text {odd }}^{2}(R)\right)=\frac{1}{2}\left(|R|-2^{s}\right)$. Observe that the functions $\left\{\mathbf{1}_{a}-\mathbf{1}_{-a}: a \in R\right\}$ span $L_{\text {odd }}^{2}(R)$, where $\mathbf{1}_{a} \in L^{2}(R)$ denotes the indicator function of the element $a \in R$. Indeed, for any $h \in L_{\text {odd }}^{2}(R)$ we have

$$
h=\frac{1}{2} \sum_{a \in R} h(a)\left(\mathbf{1}_{a}-\mathbf{1}_{-a}\right) .
$$

The dimension of $\operatorname{span}\left(\mathbf{1}_{a}-\mathbf{1}_{-a}: a \in R\right)$ is equal to one-half the number of elements $a \in R$ such that $a \neq-a$. But the elements $a \in R$ for which $a=-a$ are precisely the elements $\left(\epsilon_{1} r_{1} / 2, \ldots, \epsilon_{m} r_{m} / 2\right) \in R$ where each $\epsilon_{i}=0$ or 1 but $\epsilon_{i}=0$ for all $i$ such that $r_{i}$ is odd. That is to say, the number of elements $a \in R$ such that $a=-a$ is exactly $2^{s}$. 
We therefore conclude

$$
\begin{aligned}
\operatorname{dim} L_{\text {odd }}^{2}(R) & =\operatorname{dim} \operatorname{span}\left(\mathbf{1}_{a}-\mathbf{1}_{-a}: a \in R\right) \\
& =\frac{1}{2}\left(|R|-2^{s}\right) \\
& \leq \operatorname{dim} \operatorname{span}\left(S_{a}: a \in R\right) \\
& \leq \operatorname{dim} L_{\text {odd }}^{2}(R) .
\end{aligned}
$$

and hence equality holds throughout. Since $S_{a} \in L_{\text {odd }}^{2}(R)$ for each $a \in R$, we conclude that $L_{\text {odd }}^{2}(R)=\operatorname{span}\left(S_{a}: a \in R\right)$.

6.5. Finding a basis for each eigenspace. It therefore remains to prove Proposition 6.3. For the rest of the paper, we fix some odd $\chi \in \widehat{R^{\times}}$and let $q:=\left(q_{1}, \ldots, q_{m}\right):=q_{\chi}$ and $f:=\left(f_{1}, \ldots, f_{m}\right):=f_{\chi}$.

We start by finding an alternate representation for $w_{\chi, a}(c)$ given $a, c \in R$. This representation is based on [11, Theorem 9.9], which expresses the generalized Bernoulli number $B_{1, \chi}$ in terms of the Dirichlet $L$-function $L(s, \chi)$ evaluated at $s=1$.

Proposition 6.4. Let $a=\left(a_{1}, \ldots, a_{m}\right), c=\left(c_{1}, \ldots, c_{m}\right) \in R$. Then

$$
w_{\chi, a}(c)=\frac{i}{\pi} \sum_{k \geq 1} \frac{1}{k} \prod_{i=1}^{m}\left(\bar{\chi}_{i}^{*}\left(\frac{k a_{i} c_{i}}{\left(r_{i}, k a_{i} c_{i}\right)}\right) F_{\chi_{i}}\left(\left(r_{i}, k a_{i} c_{i}\right)\right)\right)
$$

where $F_{\chi_{i}}(\beta)=0$ if $\beta$ does not divide $q_{i}$, and otherwise

$$
F_{\chi_{i}}(\beta)=\chi_{i}^{*}\left(\frac{q_{i}}{\beta}\right) \mu\left(\frac{q_{i}}{\beta}\right) \frac{\varphi\left(r_{i}\right) \tau\left(\chi_{i}^{*}\right)}{\varphi\left(r_{i} / \beta\right)} .
$$

The factor $\tau\left(\chi_{i}^{*}\right)$ above denotes the Gauss sum of the primitive character $\chi_{i}^{*}$ :

$$
\tau\left(\chi_{i}^{*}\right):=\sum_{t \in\left(\mathbf{Z} / f_{i}\right)^{\times}} \chi_{i}^{*}(t) e\left(t / f_{i}\right) .
$$

For our purposes, the only thing we need to know about this quantity is that it is nonzero [11, Theorem 9.7].

Proof. Consider the quantity

$$
A:=\sum_{\substack{b \in R^{\times} \\ \theta_{a c b} \notin \mathbf{Z}}} \chi(b) \log \left(1-e\left(\theta_{a c b}\right)\right)
$$

where $e(x):=\exp (2 \pi i x)$, the logarithm is the principal branch, and

$$
\theta_{a c b}:=\frac{a_{1} c_{1} b_{1}}{r_{1}}+\cdots+\frac{a_{m} c_{m} b_{m}}{r_{m}} .
$$


In the sum, we replace $\log \left(1-e\left(\theta_{a c b}\right)\right)$ with its real and imaginary parts:

$$
\log \left(1-e\left(\theta_{a c b}\right)\right)=\log \left|2 \sin \left(\pi \theta_{a c b}\right)\right|+i \pi\left(\left\{\theta_{a c b}\right\}-1 / 2\right),
$$

then distribute to obtain two sums. The first of these is zero which can be seen by noting that $\left|\sin \left(\pi \theta_{a c b}\right)\right|=\left|\sin \left(\pi \theta_{-a c b}\right)\right|$ and therefore we can replace each $\chi(b)$ with $\frac{1}{2}(\chi(b)+\chi(-b))$ which is zero since $\chi$ is odd. The second sum is therefore equal to $A$, and from it we recover $w_{\chi, a}(c)$ :

$$
A=i \pi \sum_{\substack{b \in R^{\times} \\ \theta_{a c b} \notin \mathbf{Z}}} \chi(b)\left(\left\{\theta_{a c b}\right\}-1 / 2\right)=i \pi w_{\chi, a}(c) .
$$

On the other hand, we use the Taylor expansion of the logarithm to obtain

$$
A=\sum_{\substack{b \in R^{\times} \\ \theta_{a c b} \notin \mathbf{Z}}} \chi(b) \sum_{k \geq 1}-\frac{e\left(k \theta_{a c b}\right)}{k}=-\sum_{k \geq 1} \frac{1}{k} \sum_{b \in R^{\times}} \chi(b) e\left(k \theta_{a c b}\right) .
$$

Since the double sum on the left is a finite sum of convergent series, we may interchange the sums. The second equality holds since, after interchanging, the terms of the inner sum for which $\theta_{a c b} \in \mathbf{Z}$ sum to zero. Indeed, over such terms we may pull out $e\left(k \theta_{a c b}\right)=1$ and replace each $\chi(b)$ with $\frac{1}{2}(\chi(b)+\chi(-b))$ which is zero as before. We may therefore write the inner sum as the product

$$
\prod_{i=1}^{m}\left(\sum_{b_{i} \in\left(\mathbf{Z} / r_{i}\right)^{\times}} \chi_{i}\left(b_{i}\right) e\left(\frac{k a_{i} c_{i} b_{i}}{r_{i}}\right)\right) .
$$

Now let $\beta_{i, k}:=\left(r_{i}, k a_{i} c_{i}\right)$. Applying [11, Theorem 9.12], each factor above can be written

$$
\bar{\chi}_{i}^{*}\left(\frac{k a_{i} c_{i}}{\beta_{i, k}}\right) \chi_{i}^{*}\left(\frac{q_{i}}{\beta_{i, k}}\right) \mu\left(\frac{q_{i}}{\beta_{i, k}}\right) \frac{\varphi\left(r_{i}\right)}{\varphi\left(r_{i} / \beta_{i, k}\right)} \tau\left(\chi_{i}^{*}\right)
$$

if $\beta_{i, k} \mid q_{i}$. Otherwise it is zero.

Following Reid in [15, Theorem 5.16], it is more convenient to prove Proposition 6.3 by showing that the functions

$$
v_{\chi, a}:=\sum_{d \mid a} \mu(d) \bar{\chi}^{*}(d) w_{\chi, a / d}
$$

over all $a \in \mathbf{N}^{m}$ which divide $q$ are linearly independent in $L^{2}(R)$. We can accomplish this by showing that the matrix

$$
\left(v_{\chi, a}(c)\right)_{a, c}
$$


is nonsingular, where the rows and columns of the matrix are indexed by tuples $a, c \in \mathbf{N}^{m}$ such that $a \mid q$ and $c \mid q$, and $v_{\chi, a}(c):=v_{\chi, a}\left(c^{\prime}\right)$ where $c^{\prime}$ is the image of $c$ under the canonical map $\mathbf{Z}^{m} \rightarrow R$. This is done over the next three propositions. Proposition 6.5 finds an ordering of the divisors of $q$ so that:

(1) the indices $(a, c)$ of the antidiagonal entries of the matrix satisfy $a c=q$.

(2) The indices $(a, c)$ to the right of the antidiagonal entries satisfy $a c \nmid q$.

Proposition 6.6 shows that $v_{\chi, a}(c)=0$ for all $a \mid q$ and $c \mid q$ satisfying $a c \nmid q$. Finally, this paper concludes with Proposition 6.7, which shows that $v_{\chi, a}(c) \neq 0$ for all $a, c \in \mathbf{N}^{m}$ satisfying $a c=q$ and hence the matrix is indeed nonsingular.

Proposition 6.5. There exists a linear ordering

$$
a^{(1)}<a^{(2)}<\cdots<a^{(N)}
$$

of tuples in $\mathbf{N}^{m}$ which divide $q$, so that:

(1) For all $i, j=1,2, \ldots, N, i<j$ implies $a^{(j)} \nmid a^{(i)}$.

(2) For all $i=1,2, \ldots, N, a^{(i)} a^{(N-i+1)}=q$.

Proof. The tuples in $\mathbf{N}^{m}$ which divide $q$ form a graded poset with rank function given by $\operatorname{rank}\left(a_{1}, \ldots, a_{m}\right)=\sum_{i=1}^{m} \sum_{p} \nu_{p}\left(a_{i}\right)$ where the inner sum is over all primes $p$. To construct our ordering, we first specify that $a<b$ whenever $\operatorname{rank}(a)<\operatorname{rank}(b)$. Then, we arbitrarily order the elements within each level set $\operatorname{rank}^{-1}(j)$ for each $j$ in the range $0 \leq j<\operatorname{rank}(q) / 2$. If $\operatorname{rank}(q)$ is even, we further take the elements $a$ with rank equal to $\operatorname{rank}(q) / 2$ which do not satisfy $a^{2}=q$, group them into pairs of the form $(a, q / a)$, choose a unique representative from each such pair, and arbitrarily order these representatives. Next, we set $q / a>q / b$ whenever $a<b$ and $\operatorname{rank}(a)=\operatorname{rank}(b) \leq \operatorname{rank}(q) / 2$. Finally, we set $a^{((N+1) / 2)}=a$ if there exists $a$ which satisfies $a^{2}=q$. The result is a linear ordering satisfying (1) and (2).

Proposition 6.6 (c.f. [15, Proposition 5.17(i)], [8, Lemma 4.18]). If $a, c \in \mathbf{N}^{m}$ divide $q$ but $a c \nmid q$, then $v_{\chi, a}(c)=0$.

Proof. Assume $a \mid q$ and $c \mid q$ but $a c \nmid q$. Then there exists some $i \in\{1,2, \ldots, m\}, \alpha \geq 0$, and prime $p_{i}$ such that $p_{i}^{\alpha+1} \mid a_{i} c_{i}$ and $p_{i}^{\alpha} \mid q_{i}$ but $p_{i}^{\alpha+1} \nmid q_{i}$. The key insight (taken from the above two references) is that are two different possible reasons why $v_{\chi, a}(c)$ must equal zero, depending on whether or not $p_{i} \mid f_{i}$. 
First suppose $p_{i} \mid f_{i}$. Let $d \mid a$ and assume that $d_{i}$ is coprime to $f_{i}$. Then $p_{i}$ does not divide $d_{i}$ and therefore $p_{i}^{\alpha+1}$ divides $a_{i} c_{i} / d_{i}$. We also have $p_{i}^{\alpha} \mid q_{i}$ and $p_{i} \mid f_{i}$ which means $p_{i}^{\alpha+1} \mid r_{i}$. It follows that $p_{i}^{\alpha+1}$ divides $\left(r_{i}, a_{i} c_{i} / d_{i}\right)$ and hence $p_{i}^{\alpha+1} \mid\left(r_{i}, k a_{i} c_{i} / d_{i}\right)$ for every $k \geq 1$. Since $p_{i}^{\alpha+1}$ does not divide $q_{i}$, it follows that $\left(r_{i}, k a_{i} c_{i} / d_{i}\right)$ does not divide $q_{i}$ for any $k \geq 1$. By Proposition [6.4, then, we conclude $w_{\chi, a / d}(c)=0$ for every $d \mid a$ such that $d_{i}$ is coprime to $f_{i}$. But the only terms in the sum

$$
v_{\chi, a}(c)=\sum_{d \mid a} \mu(d) \bar{\chi}^{*}(d) w_{\chi, a / d}(c)
$$

which can be nonzero are the ones for which $d$ is coprime to $f$ in every component, including component $i$. This is due to the presence of the $\bar{\chi}^{*}(d)$ term which vanishes if this is not the case. It follows that $v_{\chi, a}(c)=0$ in the case $p_{i} \mid f_{i}$.

Now suppose $p_{i} \nmid f_{i}$. Since $p_{i}^{\alpha+1} \mid a_{i} c_{i}$, it follows that $p_{i}$ must divide both $a_{i}$ and $c_{i}$ since both $a_{i}$ and $c_{i}$ are divisors of $q_{i}$ and $p_{i}^{\alpha+1} \nmid q_{i}$. In particular, $p_{i}$ must divide $a_{i}$. Now let $p:=\left(1, \ldots, 1, p_{i}, 1, \ldots, 1\right)$ and let $p^{\prime}=p^{\nu p_{i}\left(a_{i}\right)}$ so that the $i^{\text {th }}$ component of $a / p^{\prime}$ is not divisible by $p_{i}$. Because the presence of the $\mu(d)$ term ensures that the sum $v_{\chi, a}(c)$ is only over $d$ with squarefree components, we can group the sum as follows:

$$
\sum_{d \mid \frac{a}{p^{\prime}}}\left(\mu(d) \bar{\chi}^{*}(d) w_{\chi, a / d}(c)+\mu(p d) \bar{\chi}^{*}(p d) w_{\chi, a / p d}(c)\right) .
$$

Since $\mu(p d)=-\mu(d)$ for every $d \mid \frac{a}{p^{\prime}}$, it suffices to show

$$
\bar{\chi}^{*}(d) w_{\chi, a / d}(c)=\bar{\chi}^{*}(p d) w_{\chi, a / p d}(c)
$$

for every $d \mid \frac{a}{p^{\prime}}$ in order to establish $v_{\chi, a}(c)=0$. By Proposition 6.4, it suffices to show that

$$
\begin{aligned}
& \bar{\chi}_{i}^{*}\left(d_{i}\right) \bar{\chi}_{i}^{*}\left(\frac{k a_{i} c_{i} / d_{i}}{\left(r_{i}, k a_{i} c_{i} / d_{i}\right)}\right) F_{\chi_{i}}\left(\left(r_{i}, k a_{i} c_{i} / d_{i}\right)\right) \\
& =\bar{\chi}_{i}^{*}\left(p_{i} d_{i}\right) \bar{\chi}_{i}^{*}\left(\frac{k a_{i} c_{i} / p_{i} d_{i}}{\left(r_{i}, k a_{i} c_{i} / p_{i} d_{i}\right)}\right) F_{\chi_{i}}\left(\left(r_{i}, k a_{i} c_{i} / p_{i} d_{i}\right)\right)
\end{aligned}
$$

for every $d \mid \frac{a}{p^{\prime}}$ and every $k \geq 1$. But since $p_{i} \nmid f_{i}$ and $p_{i} \nmid d_{i}$, we have $p_{i}^{\alpha+1} \nmid r_{i}$ while $p_{i}^{\alpha+1} \mid k a_{i} c_{i} / d_{i}$. It follows that $\left(r_{i}, k a_{i} c_{i} / d_{i}\right)=$ $\left(r_{i}, k a_{i} c_{i} / p_{i} d_{i}\right)$, and hence the above equality indeed holds for all $d \mid \frac{a}{p^{\prime}}$ and all $k \geq 1$.

Proposition 6.7 (c.f. [15, Proposition 5.17(ii)]). Let $a, c \in \mathbf{N}^{m}$ be divisors of $q$ such that $a c=q$. Then $v_{\chi, a}(c) \neq 0$. 
ON THE LINEAR SPAN OF LATTICE POINTS IN A PARALLELEPIPED 23

Proof. Suppose $d \mid a$ and each component $d_{i}$ of $d$ is squarefree and coprime to $f_{i}$. From Proposition 6.4 we can write

$$
\mu(d) \bar{\chi}^{*}(d) w_{\chi, a / d}(c)=\frac{i}{\pi} \sum_{k \geq 1} \frac{1}{k} \prod_{i=1}^{m}\left(\mu\left(d_{i}\right) \bar{\chi}_{i}^{*}\left(\frac{k q_{i}}{\beta_{i, k}}\right) F_{\chi_{i}}\left(\beta_{i, k}\right)\right)
$$

where $\beta_{i, k}:=\left(r_{i}, k q_{i} / d_{i}\right)=\left(r_{i}, k\left(a_{i} / d_{i}\right) c_{i}\right)$. Now consider the factor

$$
\mu\left(d_{i}\right) \bar{\chi}_{i}^{*}\left(\frac{k q_{i}}{\beta_{i, k}}\right) F_{\chi_{i}}\left(\beta_{i, k}\right) .
$$

which appears in the above expression. We start by showing that, regardless of whether or not $\beta_{i, k}$ divides $q_{i}$, expression (6.4) simplifies to

$$
\tau\left(\chi_{i}^{*}\right) \cdot \frac{\varphi\left(r_{i}\right)}{\varphi\left(d_{i} f_{i}\right)} \cdot \mu\left(\left(d_{i}, k\right)\right) \varphi\left(\left(d_{i}, k\right)\right) \bar{\chi}_{i}^{*}(k) .
$$

Observe that $\beta_{i, k}$ divides $q_{i}$ if and only if the last equality of

$$
\frac{q_{i}}{\beta_{i, k}}=\frac{q_{i}}{\left(r_{i}, k q_{i} / d_{i}\right)}=\frac{d_{i}}{\left(d_{i} f_{i}, k\right)}=\frac{d_{i}}{\left(d_{i}, k\right)}
$$

holds, as $d_{i}$ is coprime to $f_{i}$ by assumption. Therefore, if $\beta_{i, k} \mid q_{i}$, then plugging in $d_{i} /\left(d_{i}, k\right)$ for $q_{i} / \beta_{k, i}$ in (6.4) quickly yields (6.5). On the other hand, if $\beta_{i, k} \nmid q_{i}$, then (6.4) also simplifies to (6.5). Indeed, in this case (6.4) just equals zero since $F_{\chi_{i}}\left(\beta_{k, i}\right)$ is zero by definition. Since $\beta_{i, k} \nmid q_{i}$, the last equation in (6.6) fails to hold. This implies $k$ shares a factor with $f_{i}$, and hence $\bar{\chi}_{i}^{*}(k)=0$. So (6.5) is zero as well.

We therefore can write

$$
\mu(d) \bar{\chi}^{*}(d) w_{\chi, a / d}(c)=\frac{C_{a, \chi}^{\prime}}{\varphi(d)} \sum_{k \geq 1} \frac{\bar{\chi}(k) g_{d}(k)}{k}
$$

where:

- $C_{a, \chi}^{\prime}$ is a nonzero constant that depends only on $a$ and $\chi$

- $\bar{\chi}: \mathbf{Z} \rightarrow \mathbf{C}$ is an odd Dirichlet character defined by

$$
\bar{\chi}(k):=\prod_{i=1}^{m}{\overline{\chi_{i}}}^{*}(k)
$$

(Note: we do not put a star since this Dirichlet character may not be primitive).

- $g_{d}: \mathbf{Z} \rightarrow \mathbf{Z}$ is the function given by

$$
g_{d}(k)=\prod_{i=1}^{m} \mu\left(\left(d_{i}, k\right)\right) \varphi\left(\left(d_{i}, k\right)\right) \text {. }
$$


ON THE LINEAR SPAN OF LATTICE POINTS IN A PARALLELEPIPED 24

We now further simplify the right hand side above. Let $h_{d}: \mathbf{Z} \rightarrow \mathbf{C}$ be the function

$$
h_{d}(k)=\bar{\chi}(k)\left(\mu * g_{d}\right)(k)=\bar{\chi}(k) \sum_{\ell \mid k} \mu(\ell) g_{d}(k / \ell),
$$

where $*$ denotes Dirichlet convolution. For $k \geq 1, h_{d}(k)$ is zero unless $k$ is square-free. Indeed, if $p$ is a prime such that $p^{\alpha}$ is the highest power of $p$ dividing $k$ and $\alpha \geq 2$, then

$$
h_{d}(k)=\bar{\chi}(k) \sum_{\ell \mid \frac{k}{p^{\alpha}}}\left(\mu(\ell) g_{d}\left(\frac{k}{\ell}\right)+\mu(p \ell) g_{d}\left(\frac{k}{p \ell}\right)\right),
$$

and since $g_{d}(\ell)$ depends only on the square-free part of $\ell$, the terms in each summand cancel each other out as in (6.3). Thus we may write

$$
h_{d}(k)=\bar{\chi}(k) \sum_{\ell \mid k} \mu(k / \ell) g_{d}(\ell)=\bar{\chi}(k) \mu(k) \sum_{\ell \mid k} \mu(\ell) g_{d}(\ell) \text {. }
$$

If $n_{d}(p)$ denotes the number of indices $i \in\{1,2, \ldots, m\}$ such that $p \mid d_{i}$, then

$$
g_{d}(\ell)=\prod_{p \mid \ell}(1-p)^{n_{d}(p)}
$$

and so

$$
\begin{aligned}
\sum_{k \geq 1} \frac{h_{d}(k)}{k} & =\sum_{k \geq 1} \frac{\bar{\chi}(k) \mu(k)}{k} \prod_{p \mid k}\left(1-(1-p)^{n_{d}(p)}\right) \\
& =\prod_{p}\left(1-\frac{\bar{\chi}(p)}{p}\left(1-(1-p)^{n_{d}(p)}\right)\right)
\end{aligned}
$$

where the first product appearing above is over all primes $p$ dividing $k$, and the second product is over all primes $p$. From the first equality we see that the series on the left converges absolutely (and is in fact finite) since only finitely many primes $p$ satisfy $n_{d}(p) \geq 1$. It is a basic fact of number theory [11, Theorem 4.9] that the sum $L(1, \bar{\chi})=\sum_{k>1} \bar{\chi}(k) / k$ converges and is nonzero, and since $h_{d}=\bar{\chi}\left(\mu * g_{d}\right)$ we have $\bar{\chi} g_{d}=\bar{\chi} * h_{d}$ and therefore

$$
\sum_{k \geq 1} \frac{\bar{\chi}(k) g_{d}(k)}{k}=\left(\sum_{k \geq 1} \frac{\bar{\chi}(k)}{k}\right)\left(\sum_{k \geq 1} \frac{h_{d}(k)}{k}\right) .
$$

Moreover, since the components of $d$ are squarefree and $\varphi(p)=p-1$ for every prime $p$, we have

$$
\varphi(d)=\prod_{p}(p-1)^{n_{d}(p)}
$$


and therefore

$$
\mu(d) \bar{\chi}^{*}(d) w_{\chi, a / d}(c)=C_{a, \chi} \prod_{p} \gamma\left(p, n_{d}(p)\right)
$$

where $C_{a, \chi}$ is nonzero and depends only on $a$ and $\chi$ and

$$
\gamma(p, k):=\frac{1}{(p-1)^{k}}\left(1-\frac{\bar{\chi}(p)}{p}\right)+(-1)^{k} \frac{\bar{\chi}(p)}{p} .
$$

Now we find an expression for $v_{\chi, a}(c)$. We have

$$
v_{\chi, a}(c)=C_{a, \chi} \sum_{d \mid a^{\prime}} \prod_{p} \gamma\left(p, n_{d}(p)\right)=C_{a, \chi} \sum_{t} N(t) \prod_{p} \gamma\left(p, t_{p}\right),
$$

where the sum on the right hand side is over all tuples of nonnegative integers $t=\left(t_{2}, t_{3}, t_{5}, \ldots\right)$ indexed by the primes, $a^{\prime}=\left(a_{1}^{\prime}, \ldots, a_{m}^{\prime}\right)$ where $a_{i}^{\prime}$ is the largest squarefree divisor of $a_{i}$ coprime to $f_{i}$ for $i=$ $1,2, \ldots, m$, and $N(t)$ counts the number of $d \mid a^{\prime}$ such that $n_{d}(p)=t_{p}$ for all primes $p$. For a given tuple $t$, we have

$$
N(t)=\prod_{p}\left(\begin{array}{c}
n_{a^{\prime}}(p) \\
t_{p}
\end{array}\right)
$$

thus

$v_{\chi, a}(c)=C_{a, \chi} \sum_{t} \prod_{p}\left(\begin{array}{c}n_{a^{\prime}}(p) \\ t_{p}\end{array}\right) \gamma\left(p, t_{p}\right)=C_{a, \chi} \prod_{p}\left(\sum_{k \geq 0}\left(\begin{array}{c}n_{a^{\prime}}(p) \\ k\end{array}\right) \gamma(p, k)\right)$.

For a given prime $p$, by the binomial theorem, the inner sum is equal to 1 if $n_{a^{\prime}}(p)=0$, and otherwise equal to

$$
\left(1-\frac{\bar{\chi}(p)}{p}\right)\left(1+\frac{1}{p-1}\right)^{n_{a^{\prime}}(p)}
$$

So we conclude that

$$
v_{\chi, a}(c)=C_{a, \chi} \prod_{\substack{p \\ n_{a^{\prime}}(p) \geq 1}}\left(1-\frac{\bar{\chi}(p)}{p}\right)\left(\frac{p}{p-1}\right)^{n_{a^{\prime}}(p)} \neq 0 .
$$

\section{Acknowledgments}

This project was initiated by the author at McGill University under the supervision of Bruce Shepherd, and the author wishes to thank him for his valuable feedback and direction. The author also thanks András Sebő for his comments on this work. Finally, the author greatly appreciates the detailed suggestions and references provided by the anonymous referees. 
ON THE LINEAR SPAN OF LATTICE POINTS IN A PARALLELEPIPED 26

\section{REFERENCES}

[1] László Babai. The Fourier transform and equations over finite abelian groups. Lecture Notes, 1989.

[2] Victor Batyrev and Johannes Hofscheier. A generalization of a theorem of G. K. White. arXiv:1004.3411 [math], April 2010. arXiv: 1004.3411.

[3] Victor Batyrev and Johannes Hofscheier. Lattice polytopes, finite abelian subgroups in $\mathrm{SL}(n, \mathbf{C})$ and coding theory. arXiv:1309.5312 [math], September 2013. arXiv: 1309.5312 .

[4] Matthias Beck and Sinai Robins. Computing the Continuous Discretely: Integer-point Enumeration in Polyhedra. Springer Science \& Business Media, November 2007.

[5] Wojciech Bienia, Luis Goddyn, Pavol Gvozdjak, András Sebő, and Michael Tarsi. Flows, View Obstructions, and the Lonely Runner. Journal of Combinatorial Theory, Series B, 72(1):1-9, January 1998.

[6] Alexander Borisov. Quotient singularities, integer ratios of factorials, and the Riemann Hypothesis. International Mathematics Research Notices, 2008:rnn052, 2008.

[7] Keith Conrad. Characters of finite abelian groups. Lecture Notes, 2010.

[8] A. R. Fletcher. Inverting Reid's exact plurigenera formula. Mathematische Annalen, 284(4):617-629, December 1989.

[9] Akihiro Higashitani, Benjamin Nill, and Akiyoshi Tsuchiya. Gorenstein polytopes with trinomial $h^{*}$-polynomials. arXiv:1503.05685 [math], March 2015. arXiv: 1503.05685 .

[10] Serge Lang. Cyclotomic Fields I and II. Springer New York, January 1990.

[11] Hugh L. Montgomery and Robert C. Vaughan. Multiplicative number theory I: Classical theory, volume 97. Cambridge University Press, 2006.

[12] Shigefumi Mori. On 3-dimensional terminal singularities. Nagoya Mathematical Journal, 98:43-66, 1985.

[13] David R. Morrison and Glenn Stevens. Terminal quotient singularities in dimensions three and four. Proceedings of the American Mathematical Society, 90(1):15-20, 1984.

[14] John E. Reeve. On the volume of lattice polyhedra. Proceedings of the London Mathematical Society, 3(1):378-395, 1957.

[15] Miles Reid. Young person's guide to canonical singularities. Algebraic geometry, Bowdoin, pages 345-414, 1985.

[16] Bruce Reznick. Lattice point simplices. Discrete Mathematics, 60:219-242, June 1986.

[17] András Sebő. An introduction to empty lattice simplices. In Integer programming and combinatorial optimization, pages 400-414. Springer, 1999.

[18] Audrey Terras. Fourier Analysis on Finite Groups and Applications. Cambridge University Press, March 1999.

[19] George K. White. Lattice tetrahedra. Canad. J. Math, 16:389-396, 1964.

Department of Mathematics, Georgia Institute of Technology

E-mail address: mcelaya@gatech.edu 